\title{
Some New Fractional Estimates of Inequalities for LR- $p$-Convex Interval-Valued Functions by Means of Pseudo Order Relation
}

\author{
Muhammad Bilal Khan ${ }^{1}\left(\mathbb{D}\right.$, Pshtiwan Othman Mohammed ${ }^{2, *}\left(\mathbb{D}\right.$, Muhammad Aslam Noor ${ }^{1}$,

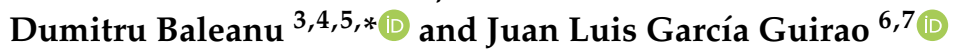 \\ 1 Department of Mathematics, COMSATS University Islamabad, Islamabad 44000, Pakistan; \\ bila142742@gmail.com (M.B.K.); noomaslam@gamil.com (M.A.N.) \\ 2 Department of Mathematics, College of Education, University of Sulaimani, \\ Sulaimani 46001, Kurdistan Region, Iraq \\ 3 Department of Mathematics, Faculty of Arts and Sciences, Cankaya University, 06530 Ankara, Turkey \\ 4 Department of Medical Research, China Medical University Hospital, China Medical University, \\ Taichung 40402, Taiwan \\ 5 Institute of Space Sciences, P.O. Box MG-23, R 76900 Magurele-Bucharest, Romania \\ 6 Departamento de Matemática Aplicada y Estadística, Campus de la Muralla, \\ Universidad Politécnica de Cartagena, 30203 Cartagena, Murcia, Spain; Juan.Garcia@upct.es \\ 7 Nonlinear Analysis and Applied Mathematics (NAAM)-Research Group, Department of Mathematics, \\ Faculty of Science, King Abdulaziz University, P.O. Box 80203, Jeddah 21589, Saudi Arabia \\ * Correspondence: pshtiwansangawi@gmail.com (P.O.M.); dumitru@cankaya.edu.tr (D.B.)
}

check for updates

Citation: Khan, M.B.; Mohammed, P.O.; Noor, M.A.; Baleanu, D.; Guirao, J.L.G. Some New Fractional Estimates of Inequalities for LR- $p$-Convex Interval-Valued Functions by Means of Pseudo Order Relation. Axioms 2021, 10, 175. https://doi.org/ 10.3390 /axioms 10030

Academic Editors: Jorge E. Macías Díaz and Chris Goodrich

Received: 28 June 2021

Accepted: 23 July 2021

Published: 31 July 2021

Publisher's Note: MDPI stays neutral with regard to jurisdictional claims in published maps and institutional affiliations.

Copyright: (c) 2021 by the authors. Licensee MDPI, Basel, Switzerland. This article is an open access article distributed under the terms and conditions of the Creative Commons Attribution (CC BY) license (https:// creativecommons.org/licenses/by/ $4.0 /)$.

\begin{abstract}
It is a familiar fact that interval analysis provides tools to deal with data uncertainty. In general, interval analysis is typically used to deal with the models whose data are composed of inaccuracies that may occur from certain kinds of measurements. In interval analysis, both the inclusion relation $(\subseteq)$ and pseudo order relation $\left(\leq_{p}\right)$ are two different concepts. In this article, by using pseudo order relation, we introduce the new class of nonconvex functions known as LR-p-convex interval-valued functions (LR-p-convex-IVFs). With the help of this relation, we establish a strong relationship between LR- $p$-convex-IVFs and Hermite-Hadamard type inequalities ( $H H$-type inequalities) via Katugampola fractional integral operator. Moreover, we have shown that our results include a wide class of new and known inequalities for LR-p-convex-IVFs and their variant forms as special cases. Useful examples that demonstrate the applicability of the theory proposed in this study are given. The concepts and techniques of this paper may be a starting point for further research in this area.
\end{abstract}

Keywords: LR-p-convex interval-valued function; Katugampola fractional integral operator; HermiteHadamard type inequality; Hermite-Hadamard-Fejér inequality

\section{Introduction}

Hermite [1] and Hadamard [2] derived the familiar inequality known as HermiteHadamard inequality ( $H H$ inequality). This inequality establishes a strong relationship with a convex function such that:

Let $f: I \rightarrow \mathbb{R}$ be a convex function defined on an interval $I \subseteq \mathbb{R}$ and $u, v \in I$ such that $v>u$. Then

$$
f\left(\frac{u+v}{2}\right) \leq \frac{1}{v-u} \int_{u}^{v} f(x) d x \leq \frac{f(u)+f(v)}{2}
$$

If $f$ is a concave function, then both inequalities are reversed. We note that $H H$-inequality may be regarded as a refinement of the concept of convexity and it follows easily from Jensen's inequality. In the last few decades, $\mathrm{HH}$-inequality has attracted many authors to devote themselves to this field. Therefore, many authors have proposed different varieties of convexities to introduce $H H$-type inequalities such as harmonic convexity [3], quasi convexity [4], Schur convexity [5,6], strong convexity [7,8], h-convexity [9], p-convexity [10], fuzzy 
convexity [11,12], fuzzy pre-invexity [13] and generalized convexity [14], $P$-convexity [15], etc. Fejér [16] considered the major generalization of $\mathrm{HH}$-inequality which is known as $H H$-Fejér inequality. It can be expressed as follows:

Let $f:[u, v] \rightarrow \mathbb{R}$ be a convex function on an interval $[u, v]$ with $u \leq v$, and let $\mathcal{W}:[u, v] \subset \mathbb{R} \rightarrow \mathbb{R}$ with $\mathcal{W} \geq 0$ be an integrable and symmetric function with respect to $\frac{u+v}{2}$. Then, we have the following inequality:

$$
f\left(\frac{u+v}{2}\right) \int_{u}^{v} \mathcal{W}(x) d x \leq \int_{u}^{v} f(x) \mathcal{W}(x) d x \leq \frac{f(u)+f(v)}{2} \int_{u}^{v} \mathcal{W}(x) d x
$$

If $f$ is concave, then the double inequality (2) is reversed. If $\mathcal{W}(x)=1$, then we obtain (1) from (2). With the assistance of inequality (2), several classical inequalities can be obtained through special convex functions. In addition, these inequalities have a very significant role for convex functions in both pure and applied mathematics. We urge the readers for a further analysis of the literature on the applications and properties of generalized convex functions and $H H$-integral inequalities, see [17-19] and the references therein.

On the other hand, it is a well-known fact that the interval-valued analysis was introduced as an attempt to overcome interval uncertainty, which occurs in the computer or mathematical models of some deterministic real-word phenomena. A classic example of an interval closure is Archimedes' technique, which is associated with the computation of the circumference of a circle. In 1966, Moore [20] gave the concept of interval analysis in his book and discussed its applications in computational Mathematics.

After that, several authors have developed a strong relationship between inequalities and IVFs by means of inclusion relation via different integral operators, as one can see by Costa [21], Costa and Roman-Flores [22], Roman-Flores et al. [23,24], and Chalco-Cano et al. [25,26], but also to more general set-valued maps by Nikodem et al. [27], and Matkowski and Nikodem [28]. In particular, Zhang et al. [29] derived the new version of Jensen's inequalities for set-valued and fuzzy set-valued functions by means of a pseudo order relation and proved that these Jensen's inequalities generalized a form of Costa Jensen's inequalities [21].

In the last two decades, in the development of pure and applied mathematics, fractional calculus has played a key role. Yet, it attains magnificent deliberation in the ongoing research work, which is due to its application in various directions such as image processing, signal processing, physics, biology, control theory, computer networking, and fluid dynamics [30-33].

As a further extension, several authors have introduced the refinements of classical inequalities through fractional integrals and discussed their applications, such as Budak et al. [34], who established a strong relationship between fractional interval $\mathrm{HH}$ inequality and convex-IVF.

Through Katugampola fractional integral [35], Toplu et al. [36] established the following $\mathrm{HH}$-inequality for $\mathrm{p}$-convex functions:

Let $f$ be a real-valued Lebesgue integrable function and $p, \alpha>0$. If $f \in S X\left([u, v], \mathbb{R}^{+}, p\right)$, then

$$
f\left(\left[\frac{u^{p}+v^{p}}{2}\right]^{\frac{1}{p}}\right) \leq \frac{p^{\alpha} \Gamma(\alpha+1)}{2\left(v^{p}-u^{p}\right)^{\alpha}}\left[\mathcal{I}_{u^{+}}^{p, \alpha} f(v)+\mathcal{I}_{v^{-}}^{p, \alpha} f(u)\right] \leq \frac{f(u)+f(v)}{2} .
$$

Due to the vast applications of convexity and fractional $\mathrm{HH}$-inequality in mathematical analysis and optimization, many authors have discussed the applications, refinements, generalizations, and extensions, see [37-56] and the references therein.

Inspired by the ongoing research work, we generalize the class of p-convex function known as LR- $p$-convex-IVF, and establish the relationship between $H H$-type inequalities and LR- $p$-convex-IVF via Katugampola fractional integral. 


\section{Preliminaries}

Let $\mathbb{R}$ be the set of real numbers and $\mathbb{R}_{I}$ be the collection of all closed and bounded intervals of $\mathbb{R}$ that is $\mathbb{R}_{I}=\{[\underline{\xi}, \bar{\xi}]: \underline{\xi}, \bar{\xi} \in \mathbb{R}$ and $\underline{\xi} \leq \bar{\xi}\}$. If $\underline{\xi} \geq 0$, then $[\underline{\xi}, \bar{\xi}]$ is called positive interval. The set of all positive intervals is denoted by $\mathbb{R}_{I}^{+}$and defined as

$$
\mathbb{R}_{I}^{+}=\left\{[\underline{\xi}, \bar{\xi}]:[\underline{\xi}, \bar{\xi}] \in \mathbb{R}_{I} \text { and } \underline{\xi} \geq 0\right\}
$$

Let $\varrho \in \mathbb{R}$ and $\varrho \xi$ be defined as

$$
\varrho \xi=\left\{\begin{array}{c}
{[\varrho \underline{\xi}, \varrho \bar{z}], \varrho>0} \\
\{0\}, \varrho=0 \\
{[\varrho \bar{\xi}, \varrho \underline{z}], \varrho<0 .}
\end{array}\right.
$$

Then, the addition $\xi_{1}+\xi_{2}$ and Minkowski difference $\xi_{1}-\xi_{2}$ for $\xi_{1}, \xi_{2} \in \mathbb{R}_{I}$ are defined by

$$
\xi_{1}+\xi_{2}=\left[\underline{\xi}_{1}, \bar{\xi}_{1}\right]+\left[\underline{\xi}_{2}, \bar{\xi}_{2}\right]=\left[\underline{\xi}_{1}+\underline{\xi}_{2}, \bar{\xi}_{1}+\bar{\xi}_{2}\right]
$$

and

$$
\xi_{1}-\xi_{2}=\left[\underline{\xi}_{1}, \bar{\xi}_{1}\right]-\left[\underline{\xi}_{2}, \bar{\xi}_{2}\right]=\left[\underline{\xi}_{1}-\bar{\xi}_{2}, \bar{\xi}_{1}-\underline{\xi}_{2}\right]
$$

respectively.

The inclusion relation " $\supseteq$ " means that

$$
\xi_{2} \supseteq \xi_{1} \Leftrightarrow\left[\underline{\xi}_{2}, \bar{\xi}_{2}\right] \supseteq\left[\underline{\xi}_{1}, \bar{\xi}_{1}\right] \Leftrightarrow\left[\underline{\xi}_{1} \geq \underline{\xi}_{2}, \bar{\xi}_{2} \geq \bar{\xi}_{1}\right]
$$

Remark 1. ([29]). (i) The relation " $\leq_{p}$ " defined on $\mathbb{R}_{I}$ by

$$
[\underline{\xi}, \bar{\xi}] \leq p[\underline{\zeta}, \bar{\zeta}] \text { if and only if } \underline{\xi} \leq \underline{\zeta}, \bar{\xi} \leq \bar{\zeta}
$$

for all $[\underline{\xi}, \bar{\xi}],[\underline{\zeta}, \bar{\zeta}] \in \mathbb{R}_{I}$ is a pseudo order relation. In the interval analysis case, both the pseudo order relation $\left(\leq_{p}\right)$ and partial order relation $(\leq)$ behave alike, thus the relation $[\underline{\xi}, \bar{\xi}] \leq_{p}[\underline{\zeta}, \bar{\zeta}]$ is coincident to $[\underline{\xi}, \bar{\xi}] \leq[\underline{\zeta}, \bar{\zeta}]$ on $\mathbb{R}_{I}$, for more details see, [21,29].

(ii) It can be easily seen that " $\leq p$ " looks similar to "left and right" on the real line $\mathbb{R}$, so we call " $\leq_{p}$ " is "left and right" (or "LR" order, in short).

The concept of Riemann integral for IVF first introduced by Moore [20] is defined as follows:

Theorem 1. ([20]). Let $f:[u, v] \subset \mathbb{R} \rightarrow \mathbb{R}_{I}$ is an IVF such that $f(x)=[\underline{f}(x), \bar{f}(x)]$. Then, $f$ is Riemann integrable over $[u, v]$ if and only if, $f$ and $\bar{f}$ both are Riemann integrable over $[u, v]$ such that

$$
(I R) \int_{u}^{v} f(x) d x=\left[(R) \int_{u}^{v} \underline{f}(x) d x,(R) \int_{u}^{v} \bar{f}(x) d x\right]
$$

Now, we discuss the concept of Katugampola fractional integral operator for IVF.

Let $q \geq 1, c \in \mathbb{R}$ and $x_{c}^{q}(u, v)$ be the set of all complex-valued Lebesgue integrable IVFs $f$ on $[u, v]$ for which the norm $\|f\| \mathcal{X}_{c}^{q}$ is defined by

$$
\|f\| \mathcal{X}_{c}^{q}=\left(\int_{u}^{v}\left|\varrho^{c} f(x)\right|^{q} \frac{d \varrho}{\varrho}\right)^{\frac{1}{q}}<\infty
$$


For $1 \leq q<\infty$ and

$$
\|f\| \mathcal{X}_{c}^{\infty}=e s s \sup _{u \leq \varrho \leq \nu} \varrho^{c}|f(\varrho)|
$$

Katugampola [35] presented a new fractional integral to generalize the Riemann Liouville and Hadamard fractional integrals under certain conditions.

Let $p, \alpha>0$ and $f \in \Im \mathcal{L}_{[u, v]}$ be the collection of all complex-valued Lebesgue integrable IVFs on $[u, v]$. Then, the interval left and right Katugampola fractional integrals of $f \in \Im \mathcal{L}_{[u, v]}$ with order are defined by

$$
\mathcal{I}_{u^{+}}^{p, \alpha} f(x)=\frac{p^{1-\alpha}}{\Gamma(\alpha)} \int_{u}^{x}\left(x^{p}-\zeta^{p}\right)^{\alpha-1} \zeta^{p-1} f(\zeta) d(\zeta)(x>u),
$$

and

$$
\mathcal{I}_{v^{-}}^{p, \alpha} f(x)=\frac{p^{1-\alpha}}{\Gamma(\alpha)} \int_{x}^{v}\left(\zeta^{p}-x^{p}\right)^{\alpha-1} \zeta^{p-1} f(\zeta) d(\zeta)(x<v)
$$

respectively, where $\Gamma(x)=\int_{0}^{\infty} \zeta^{x-1} u^{-\zeta} d(\zeta)$ is the Euler gamma function.

The concept of $p$-convex functions were established by Zhang and Wang [10], and a number of properties of the functions were introduced.

Definition 1. ([54]). Let $p \in \mathbb{R}$ with $p \neq 0$. Then, the interval I is said to be $p$-convex if

$$
\left[\varrho x^{p}+(1-\varrho) y^{p}\right]^{\frac{1}{p}} \in I,
$$

for all $x, y \in I, \varrho \in[0,1]$, where $p=2 n+1$ and $n \in N$ or $p$ is an odd number.

Definition 2. ([10]). Let $p \in \mathbb{R}$ with $p \neq 0$ and $I=[u, v] \subseteq \mathbb{R}$. Then, the function $f:[u, v] \rightarrow \mathbb{R}^{+}$is said to be $p$-convex function if

$$
f\left(\left[\varrho x^{p}+(1-\varrho) y^{p}\right]^{\frac{1}{p}}\right) \leq \varrho f(x)+(1-\varrho) f(y),
$$

for all $x, y \in[u, v], \varrho \in[0,1]$. If the inequality (13) is reversed, then $f$ is called $p$-concave function. The set of all $p$-convex (LR-p-concave, $L R$-p-affine) functions is denoted by

$$
S X\left([u, v], \mathbb{R}^{+}, p\right)\left(S V\left([u, v], \mathbb{R}^{+}, p\right)\right) .
$$

Firstly, we introduce the new class of LR-p-convex-IVF.

\section{LR- $p$-Convex Interval-Valued Functions}

Now, we introduce LR- $p$-convex interval-valued functions.

Definition 3. The IVF $f:[u, v] \rightarrow \mathbb{R}_{I}^{+}$is said to be LR-p-convex-IVF if for all $x, y \in[u, v]$ and $\varrho \in[0,1]$ we have

$$
f\left(\left[\varrho x^{p}+(1-\varrho) y^{p}\right]^{\frac{1}{p}}\right) \leq_{p} \varrho f(x)+(1-\varrho) f(y) .
$$

If inequality (14) is reversed, then $f$ is said to be LR-p-concave on $[u, v]$. The set of all LR- $p$-convex (LR- $p$-concave) IVFs is denoted by

$$
\operatorname{LRSX}\left([u, v], \mathbb{R}_{I}^{+}, p\right)\left(\operatorname{LRSV}\left([u, v], \mathbb{R}_{I}^{+}, p\right)\right) .
$$

Remark 2. If $p=1$, then LR-p-convex-IVF reduces to LR-convex-IVF, see [24]. 
If $p=-1$, then we obtain the class of harmonically convex functions, which is also a new one.

The next Theorem 2 establishes the relationship between Definition 3 and end point functions of IVFs.

Theorem 2. Let $f:[u, v] \rightarrow \mathbb{R}_{I}^{+}$be an IVF defined by $f(x)=[\underline{f}(x), \bar{f}(x)]$, for all $x \in[u, v]$. Then, $f \in \operatorname{LRSX}\left([u, v], \mathbb{R}_{I}^{+}, p\right)$ if and only if, $\underline{f}, \bar{f} \in S X\left([u, v], \mathbb{R}^{+}, p\right)$.

Proof. Assume that $f, \bar{f} \in S X\left([u, v], \mathbb{R}^{+}, p\right)$. Then, for all $x, y \in[u, v], \varrho \in[0,1]$, we have

$$
\underline{f}\left(\left[\varrho x^{p}+(1-\varrho) y^{p}\right]^{\frac{1}{p}}\right) \leq \varrho \underline{f}(x)+(1-\varrho) \underline{f}(y)
$$

and

$$
\bar{f}\left(\left[\varrho x^{p}+(1-\varrho) y^{p}\right]^{\frac{1}{p}}\right) \leq \varrho \bar{f}(x)+(1-\varrho) \bar{f}(y)
$$

From Definition 3 and order relation $\leq_{p}$, we have

$$
\begin{gathered}
{\left[\underline{f}\left(\left[\varrho x^{p}+(1-\varrho) y^{p}\right]^{\frac{1}{p}}\right), \bar{f}\left(\left[\varrho x^{p}+(1-\varrho) y^{p}\right]^{\frac{1}{p}}\right)\right]} \\
\leq p[\varrho \underline{f}(x)+(1-\varrho) \underline{f}(y), \varrho \bar{f}(x)+(1-\varrho) \bar{f}(y)] \\
\quad=\varrho[\underline{f}(x), \bar{f}(x)]+(1-\varrho)[\underline{f}(y), \bar{f}(y)]
\end{gathered}
$$

That is

$$
f\left(\left[\varrho x^{p}+(1-\varrho) y^{p}\right]^{\frac{1}{p}}\right) \leq_{p} \varrho f(x)+(1-\varrho) f(y), \forall x, y \in[u, v], \varrho \in[0,1] .
$$

Hence, $f \in \operatorname{LRSX}\left([u, v], \mathbb{R}_{I}^{+}, p\right)$. have

Conversely, let $f \in \operatorname{LRSX}\left([u, v], \mathbb{R}_{I}^{+}, p\right)$. Then, for all $x, y \in[u, v]$ and $\varrho \in[0,1]$, we

$$
f\left(\left[\varrho x^{p}+(1-\varrho) y^{p}\right]^{\frac{1}{p}}\right) \leq_{p} \varrho f(x)+(1-\varrho) f(y) .
$$

That is

$$
\begin{gathered}
{\left[\underline{f}\left(\left[\varrho x^{p}+(1-\varrho) y^{p}\right]^{\frac{1}{p}}\right), \bar{f}\left(\left[\varrho x^{p}+(1-\varrho) y^{p}\right]^{\frac{1}{p}}\right)\right] \leq_{p} \varrho[\underline{f}(x), \bar{f}(x)]+(1-\varrho)[\underline{f}(y), \bar{f}(y)]} \\
=[\varrho \underline{f}(x)+(1-\varrho) \underline{f}(y), \varrho \bar{f}(x)+(1-\varrho) \bar{f}(y)]
\end{gathered}
$$

It follows that

$$
\underline{f}\left(\left[\varrho x^{p}+(1-\varrho) y^{p}\right]^{\frac{1}{p}}\right) \leq \varrho \underline{f}(x)+(1-\varrho) \underline{f}(y),
$$

and

$$
\bar{f}\left(\left[\varrho x^{p}+(1-\varrho) y^{p}\right]^{\frac{1}{p}}\right) \leq \varrho \bar{f}(x)+(1-\varrho) \bar{f}(y),
$$

Hence, the result follows.

Remark 3. If $\underline{f}(x)=\bar{f}(x)$, then $p$-convex-IVF reduces to the classical p-convex function, see [10].

If $f(x)=\bar{f}(x)$ with $\gamma=1$ and $p=1$, then $p$-convex-IVF reduces to the classical convex function. 
Example 1. Let $p$ be an odd number, $\alpha=\frac{1}{2}, x \in[2,3]$ and $f(x)=\left[-x^{\frac{p}{2}}, 2-x^{\frac{p}{2}}\right]$. Then, we clearly see that both end point functions $f(x)=-x^{\frac{p}{2}}$ and $\bar{f}(x)=2-x^{\frac{p}{2}}$ are $p$-convex functions. Hence, $f \in \operatorname{LRSX}\left([u, v], \mathbb{R}_{I}^{+}, p\right)$.

\section{Fractional Hermite-Hadamard Type Inequalities}

In this section, we will prove some new Hermite-Hadamard type inequalities for LR- $p$-convex-IVFs by means of the pseudo order relation via Katugampola fractional integral operator.

Theorem 3. Let $p, \alpha>0, u, v \in I$ such that $v>u, f \in \Im \mathcal{L}_{([u, v]]}$. If $f \in \operatorname{LRSX}\left([u, v], \mathbb{R}_{I}^{+}, p\right)$, then

$$
f\left(\left[\frac{u^{p}+v^{p}}{2}\right]^{\frac{1}{p}}\right) \leq_{p} \frac{p^{\alpha} \Gamma(\alpha+1)}{2\left(v^{p}-u^{p}\right)^{\alpha}}\left[\mathcal{I}_{u^{+}}^{p, \alpha} f(v)+\mathcal{I}_{v^{-}}^{p, \alpha} f(u)\right] \leq_{p} \frac{f(u)+f(v)}{2} .
$$

If $f \in \operatorname{LRSV}\left([u, v], \mathbb{R}_{I}^{+}, p\right)\left([u, v], \mathbb{R}_{I}^{+}, p\right)$, then

$$
f\left(\left[\frac{u^{p}+v^{p}}{2}\right]^{\frac{1}{p}}\right) \geq_{p} \frac{p^{\alpha} \Gamma(\alpha+1)}{2\left(v^{p}-u^{p}\right)^{\alpha}}\left[\mathcal{I}_{u^{+}}^{p, \alpha} f(v)+\mathcal{I}_{v^{-}}^{p, \alpha} f(u)\right] \geq_{p} \frac{f(u)+f(v)}{2} .
$$

Proof. Let $f \in \operatorname{LRSX}\left([u, v], \mathbb{R}_{I}^{+}, p\right)$. Then, by hypothesis, we have

$$
2 f\left(\left[\frac{u^{p}+v^{p}}{2}\right]^{\frac{1}{p}}\right) \leq_{p} f\left(\left[\varrho u^{p}+(1-\varrho) v^{p}\right]^{\frac{1}{p}}\right)+f\left(\left[(1-\varrho) u^{p}+\varrho v^{p}\right]^{\frac{1}{p}}\right)
$$

Multiplying both sides (17) by $\varrho^{\alpha-1}$ and integrating the obtained result with respect to $\varrho$ over $(0,1)$, we have

$$
\begin{gathered}
2 \int_{0}^{1} \varrho^{\alpha-1} f\left(\left[\frac{u^{p}+v^{p}}{2}\right]^{\frac{1}{p}}\right) d \varrho \\
\leq p \int_{0}^{1} \varrho^{\alpha-1}\left[f\left(\left[\varrho u^{p}+(1-\varrho) \nu^{p}\right]^{\frac{1}{p}}\right)+f\left(\left[(1-\varrho) u^{p}+\varrho v^{p}\right]^{\frac{1}{p}}\right)\right] d \varrho
\end{gathered}
$$

From (18), we get

$$
\begin{aligned}
& 2 \int_{0}^{1} \varrho^{\alpha-1} f\left(\left[\frac{u^{p}+v^{p}}{2}\right]^{\frac{1}{p}}\right) d \varrho=2\left[\int_{0}^{1} \varrho^{\alpha-1} \underline{f}\left(\left[\frac{u^{p}+v^{p}}{2}\right]^{\frac{1}{p}}\right) d \varrho, \int_{0}^{1} \varrho^{\alpha-1} \bar{f}\left(\left[\frac{u^{p}+v^{p}}{2}\right]^{\frac{1}{p}}\right) d \varrho\right] \\
& =2\left[\frac{1}{\alpha} f\left(\left[\frac{u^{p}+v^{p}}{2}\right]^{\frac{1}{p}}\right), \frac{1}{\alpha} \bar{f}\left(\left[\frac{u^{p}+v^{p}}{2}\right]^{\frac{1}{p}}\right)\right] \\
& =2 \frac{1}{\alpha} f\left(\left[\frac{u^{p}+v^{p}}{2}\right]^{\frac{1}{p}}\right) \text {. }
\end{aligned}
$$

and

$$
\begin{aligned}
& \int_{0}^{1} \varrho^{\alpha-1}\left[f\left(\left[\varrho u^{p}+(1-\varrho) v^{p}\right]^{\frac{1}{p}}\right)+f\left(\left[(1-\varrho) u^{p}+\varrho v^{p}\right]^{\frac{1}{p}}\right)\right] d \varrho \\
& =\int_{0}^{1} \varrho^{\alpha-1}\left[f\left(\left[\varrho u^{p}+(1-\varrho) v^{p}\right]^{\frac{1}{p}}\right), \underline{f}\left(\left[\varrho u^{p}+(1-\varrho) v^{p}\right]^{\frac{1}{p}}\right)\right] d \varrho \\
& +\int_{0}^{1} \varrho^{\alpha-1}\left[\bar{f}\left(\left[(1-\varrho) u^{p}+\varrho v^{p}\right]^{\frac{1}{p}}\right), \bar{f}\left(\left[(1-\varrho) u^{p}+\varrho v^{p}\right]^{\frac{1}{p}}\right)\right] d \varrho
\end{aligned}
$$


Let $\varrho \in[0,1], x^{p}=\varrho u^{p}+(1-\varrho) v^{p}$ and $y^{p}=(1-\varrho) u^{p}+\varrho v^{p}$. Then, we have

$$
\begin{gathered}
=\frac{p}{\left(v^{p}-u^{p}\right)^{\alpha}}\left[\int_{u}^{v}\left(v^{p}-y^{p}\right)^{\alpha-1} \frac{f(y)}{y^{1-p}} d y, \int_{u}^{v}\left(v^{p}-y^{p}\right)^{\alpha-1} \frac{\bar{f}(y)}{y^{1-p}} d y\right] \\
+\frac{p}{\left(v^{p}-u^{p}\right)^{\alpha}}\left[\int_{u}^{v}\left(x^{p}-u^{p}\right)^{\alpha-1} \frac{f(x)}{\bar{x}^{1-p}} d x, \int_{u}^{v}\left(x^{p}-u^{p}\right)^{\alpha-1} \frac{\bar{f}(x)}{x^{1-p}} d x\right], \\
=\frac{p}{\left(v^{p}-u^{p}\right)^{\alpha}}\left[\int_{u}^{v}\left(v^{p}-u^{p}\right)^{\alpha-1} \frac{f(y)}{y^{1-p}} d y, \int_{u}^{v}\left(x^{p}-u^{p}\right)^{\alpha-1} \frac{f(x)}{x^{1-p}} d x\right], \\
\quad \leq_{p} \frac{p^{\alpha} \Gamma(\alpha)}{\left(v^{p}-u^{p}\right)^{\alpha}}\left[\mathcal{I}_{u^{+}}^{p, \alpha} f(v)+\mathcal{I}_{v^{-}}^{p, \alpha} f(u)\right] .
\end{gathered}
$$

Since $f \in \operatorname{LRSX}\left([u, v], \mathbb{R}_{I}^{+}, p\right)$, we obtain

$$
f\left(\left[\varrho u^{p}+(1-\varrho) v^{p}\right]^{\frac{1}{p}}\right) \leq_{p} \varrho f(u)+(1-\varrho) f(v)
$$

and

$$
f\left(\left[\varrho v^{p}+(1-\varrho) u^{p}\right]^{\frac{1}{p}}\right) \leq_{p} \varrho f(v)+(1-\varrho) f(u)
$$

Adding (21) and (22), we get

$$
f\left(\left[\varrho u^{p}+(1-\varrho) v^{p}\right]^{\frac{1}{p}}\right)+f\left(\left[\varrho v^{p}+(1-\varrho) u^{p}\right]^{\frac{1}{p}}\right) \leq_{p} f(u)+f(v)
$$

Multiplying both sides (23) by $\varrho^{\alpha-1}$ and integrating both sides of the obtained result with respect to $\varrho$ over $(0,1)$, we get

$$
\frac{p^{\alpha} \Gamma(\alpha)}{\left(v^{p}-u^{p}\right)^{\alpha}}\left[\mathcal{I}_{u^{+}}^{p, \alpha} f(v)+\mathcal{I}_{v^{-}}^{p, \alpha} f(u)\right] \leq_{p} \frac{f(u)+f(v)}{\alpha}
$$

From (20) and (24), (19) becomes

$$
f\left(\left[\frac{u^{p}+v^{p}}{2}\right]^{\frac{1}{p}}\right) \leq_{p} \frac{p^{\alpha} \Gamma(\alpha+1)}{2\left(v^{p}-u^{p}\right)^{\alpha}}\left[\mathcal{I}_{u^{+}}^{p, \alpha} f(v)+\mathcal{I}_{v^{-}}^{p, \alpha} f(u)\right] \leq_{p} \frac{f(u)+f(v)}{2},
$$

and the theorem has been proved.

Remark 4. Let $p=1$. Then, Theorem 3 reduces to the result for LR-convex-IVF, which is also a new one:

$$
f\left(\frac{u+v}{2}\right) \leq_{p} \frac{\Gamma(\alpha+1)}{2(v-u)^{\alpha}}\left[\mathcal{I}_{u^{+}}^{\alpha} f(v)+\mathcal{I}_{v^{-}}^{\alpha} f(u)\right] \leq_{p} \frac{f(u)+f(v)}{2} .
$$

If $\alpha=1$, then Theorem 3 reduces to the result for LR- $p$-convex-IVF, which is also a new one:

$$
f\left(\left[\frac{u^{p}+v^{p}}{2}\right]^{\frac{1}{p}}\right) \leq_{p} \frac{p}{v^{p}-u^{p}}(I R) \int_{u}^{v} x^{p-1} f(x) d x \leq_{p} \frac{f(u)+f(v)}{2}
$$

Let $p=\alpha=1$. Then, Theorem 3 reduces to the result for LR-p-convex-IVF, which is also a new one:

$$
f\left(\frac{u+v}{2}\right) \leq_{p} \frac{1}{v-u}(I R) \int_{u}^{v} f(x) d x \leq_{p} \frac{f(u)+f(v)}{2}
$$

If $\underline{f}=\bar{f}$, then we get inequality (13) from Theorem 3 . 
If $p=1$ and $\underline{f}=\bar{f}$, then from Theorem 3, we obtain fractional $H H$-inequality for convex function, see [41]:

$$
f\left(\frac{u+v}{2}\right) \leq \frac{\Gamma(\alpha+1)}{2(v-u)^{\alpha}}\left[\mathcal{I}_{u^{+}}^{\alpha} f(v)+\mathcal{I}_{v^{-}}^{\alpha} f(u)\right] \leq \frac{f(u)+f(v)}{2} .
$$

If $\alpha=1$, and $f=\bar{f}$, then Theorem 3 reduces to the result for LR-p-convex-IVF, see [10]:

$$
f\left(\left[\frac{u^{p}+v^{p}}{2}\right]^{\frac{1}{p}}\right) \leq \frac{p}{v^{p}-u^{p}} \int_{u}^{v} x^{p-1} f(x) d x \leq \frac{f(u)+f(v)}{2}
$$

If $\alpha=p=1$ and $f=\bar{f}$, then we obtain the classical inequality (1) from Theorem 3 .

Example 2. Let $p$ be an odd number, $\alpha=\frac{1}{2}, x \in[2,3]$ and $f(x)=\left[2-x^{\frac{p}{2}}, 2\left(2-x^{\frac{p}{2}}\right)\right]$. Then, we clearly see that $f \in \Im \mathcal{L}_{([u, v])}$ and $f \in \operatorname{LRSX}\left([u, v], \mathbb{R}_{I}^{+}, p\right)$. Since $f(x)=2-x^{\frac{p}{2}}$ and $\bar{f}(x)=2\left(2-x^{\frac{p}{2}}\right)$. Now, we compute the following:

$$
\begin{aligned}
\underline{f}\left(\left[\frac{u^{p}+v^{p}}{2}\right]^{\frac{1}{p}}\right) & =\underline{f}\left(\frac{5}{2}\right)=\frac{4-\sqrt{10}}{2} \\
\bar{f}\left(\left[\frac{u^{p}+v^{p}}{2}\right]^{\frac{1}{p}}\right) & =\bar{f}\left(\frac{5}{2}\right)=4-\sqrt{10}, \\
\frac{f(u)+f(v)}{2} & =2-\frac{\sqrt{2}-\sqrt{3}}{2}, \\
\frac{\bar{f}(u)+\bar{f}(v)}{2} & =4-\sqrt{2}-\sqrt{3} .
\end{aligned}
$$

Note that

$$
\begin{gathered}
\frac{p^{\alpha} \Gamma(\alpha+1)}{2\left(v^{p}-u^{p}\right)^{\alpha}}\left[\mathcal{I}_{u^{+}}^{p, \alpha} \underline{f}(v)+\mathcal{I}_{v^{-}}^{p, \alpha} \underline{f}(u)\right]=\frac{\Gamma\left(\frac{3}{2}\right)}{2} \frac{1}{\sqrt{\pi}} \int_{2}^{3}\left(3^{p}-x^{p}\right)^{\frac{-1}{2}} x^{p-1}\left[2-x^{\frac{p}{2}}, 2\left(2-x^{\frac{p}{2}}\right)\right] d x \\
+\frac{\Gamma\left(\frac{3}{2}\right)}{2} \frac{1}{\sqrt{\pi}} \int_{2}^{3}\left(x^{p}-2^{p}\right)^{\frac{-1}{2}} x^{p-1}\left[2-x^{\frac{p}{2}}, 2\left(2-x^{\frac{p}{2}}\right)\right] d x \\
=\frac{1}{4}\left[\frac{7393}{5000}+\frac{9501}{5000}\right]=\frac{8447}{10,000}
\end{gathered}
$$

and

$$
\begin{gathered}
\frac{p^{\alpha} \Gamma(\alpha)}{\left(v^{p}-u^{p}\right)^{\alpha}}\left[\mathcal{I}_{u^{+}}^{p, \alpha} \bar{f}(v)+\mathcal{I}_{v^{-}}^{p, \alpha} \bar{f}(u)\right]=\frac{\Gamma\left(\frac{3}{2}\right)}{2} \frac{1}{\sqrt{\pi}} \int_{2}^{3}\left(3^{p}-x^{p}\right)^{\frac{-1}{2}} x^{p-1}\left(2-x^{\frac{p}{2}}\right) d x \\
+\frac{\Gamma\left(\frac{3}{2}\right)}{2} \frac{1}{\sqrt{\pi}} \int_{2}^{3}\left(x^{p}-2^{p}\right)^{\frac{-1}{2}} x^{p-1}\left(2-x^{\frac{p}{2}}\right) d x \\
=\frac{1}{4}\left[\frac{7393}{10,000}+\frac{9501}{10,000}\right]=\frac{8447}{20,000} .
\end{gathered}
$$

Therefore, we have

$$
\begin{aligned}
& \frac{4-\sqrt{10}}{2} \leq \frac{8447}{20,000} \leq 2-\frac{\sqrt{2}+\sqrt{3}}{2} \\
& 4-\sqrt{10} \leq \frac{8447}{10,000} \leq 4-\sqrt{2}-\sqrt{3}
\end{aligned}
$$

and Theorem 3 is verified.

The next Theorem 4 gives the HH-Fejér type inequality for LR-p-convex-IVFs. 
Theorem 4. Let $p, \alpha>0, u, v \in I$ with $v>u, f \in \Im \mathcal{L}_{([u, v])}$ and $\mathcal{W}(x)=\mathcal{W}\left(\left[u^{p}+v^{p}-x^{p}\right]^{\frac{1}{p}}\right)$ $\geq 0$ for $x \in I$. If $f \in \operatorname{LRSX}\left([u, v], \mathbb{R}_{I}^{+}, p\right)$, then we have the HH-Fejér type inequality as follows:

$$
\begin{aligned}
& f\left(\left[\frac{u^{p}+v^{p}}{2}\right]^{\frac{1}{p}}\right)\left[\mathcal{I}_{u^{+}}^{p, \alpha} \mathcal{W}(v)+\mathcal{I}_{v^{-}}^{p, \alpha} \mathcal{W}(u)\right] \\
& \leq p\left[\mathcal{I}_{u^{+}}^{p, \alpha} \mathcal{W}(v)+\mathcal{I}_{v^{-}}^{p, \alpha} f \mathcal{W}(u)\right] \leq_{p} \frac{f(u)+f(v)}{2}\left[\mathcal{I}_{u^{+}}^{p, \alpha} \mathcal{W}(v)+\mathcal{I}_{v^{-}}^{p, \alpha} \mathcal{W}(u)\right] .
\end{aligned}
$$

If $f \in \operatorname{LRSV}\left([u, v], \mathbb{R}_{I}^{+}, p\right)$, then

$$
\begin{gathered}
f\left(\left[\frac{u^{p}+v^{p}}{2}\right]^{\frac{1}{p}}\right)\left[\mathcal{I}_{u^{+}}^{p, \alpha} \mathcal{W}(v)+\mathcal{I}_{v^{-}}^{p, \alpha} \mathcal{W}(u)\right] \geq_{p}\left[\mathcal{I}_{u^{+}}^{p, \alpha} f \mathcal{W}(v)+\mathcal{I}_{v^{-}}^{p, \alpha} f \mathcal{W}(u)\right] \\
\geq_{p} \frac{f(u)+f(v)}{2}\left[\mathcal{I}_{u^{+}}^{p, \alpha} \mathcal{W}(v)+\mathcal{I}_{v^{-}}^{p, \alpha} \mathcal{W}(u)\right] .
\end{gathered}
$$

Proof. Since $f \in \operatorname{LRSX}\left([u, v], \mathbb{R}_{I}^{+}, p\right)$, then for $\varrho \in[0,1]$, we have

$$
f\left(\left[\frac{u^{p}+v^{p}}{2}\right]^{\frac{1}{p}}\right) \leq_{p} \frac{1}{2}\left(f\left(\left[\varrho u^{p}+(1-\varrho) v^{p}\right]^{\frac{1}{p}}\right)+f\left(\left[(1-\varrho) u^{p}+\varrho v^{p}\right]^{\frac{1}{p}}\right)\right) .
$$

Since $\mathcal{W}\left(\left[\varrho u^{p}+(1-\varrho) v^{p}\right]^{\frac{1}{p}}\right)=\mathcal{W}\left(\left[\varrho v^{p}+(1-\varrho) u^{p}\right]^{\frac{1}{p}}\right)$, then multiplying both sides of (27) by $\varrho^{\alpha-1} \mathcal{W}\left(\left[(1-\varrho) u^{p}+\varrho v^{p}\right]^{\frac{1}{p}}\right)$, and integrating it with respect to $\varrho$ over $[0,1]$, we have

$$
\begin{aligned}
& 2 \int_{0}^{1} \varrho^{\alpha-1} f\left(\left[\frac{u^{p}+v^{p}}{2}\right]^{\frac{1}{p}}\right) \mathcal{W}\left(\left[(1-\varrho) u^{p}+\varrho v^{p}\right]^{\frac{1}{p}}\right) d \varrho \\
& \leq p \int_{0}^{1} \varrho^{\alpha-1} f\left(\left[\varrho u^{p}+(1-\varrho) v^{p}\right]^{\frac{1}{p}}\right) \mathcal{W}\left(\left[(1-\varrho) u^{p}+\varrho v^{p}\right]^{\frac{1}{p}}\right) d \varrho \\
& +\int_{0}^{1} \varrho^{\alpha-1} f\left(\left[(1-\varrho) u^{p}+\varrho v^{p}\right]^{\frac{1}{p}}\right) \mathcal{W}\left(\left[(1-\varrho) u^{p}+\varrho v^{p}\right]^{\frac{1}{p}}\right) d \varrho \\
& =\int_{0}^{1} \varrho^{\alpha-1}\left[\underline{f}\left(\left[\varrho u^{p}+(1-\varrho) v^{p}\right]^{\frac{1}{p}}\right), \bar{f}\left(\left[(1-\varrho) u^{p}+\varrho v^{p}\right]^{\frac{1}{p}}\right)\right] \times \mathcal{W}\left(\left[(1-\varrho) u^{p}+\varrho v^{p}\right]^{\frac{1}{p}}\right) d \varrho+ \\
& \int_{0}^{1} \varrho^{\alpha-1}\left[\underline{f}\left(\left[(1-\varrho) u^{p}+\varrho v^{p}\right]^{\frac{1}{p}}\right), \bar{f}\left(\left[(1-\varrho) u^{p}+\varrho v^{p}\right]^{\frac{1}{p}}\right)\right] \times \mathcal{W}\left(\left[(1-\varrho) u^{p}+\varrho v^{p}\right]^{\frac{1}{p}}\right) d \varrho .
\end{aligned}
$$


Let $x^{p}=\varrho v^{p}+(1-\varrho) u^{p}$. Then, we have

$$
\begin{aligned}
& \frac{2 p}{\left(v^{p}-u^{p}\right)^{\alpha}} f\left(\left[\frac{u^{p}+v^{p}}{2}\right]^{\frac{1}{p}}\right) \int_{u}^{v}\left(x^{p}-u^{p}\right)^{\alpha-1} \mathcal{W}(x) d x \\
& \leq p \frac{p}{\left(v^{p}-u^{p}\right)^{\alpha}} \int_{u}^{v}\left(x^{p}-u^{p}\right)^{\alpha-1}\left[\underline{f}\left(\left[u^{p}-v^{p}-x^{p}\right]^{\frac{1}{p}}\right), \bar{f}\left(\left[u^{p}+v^{p}-x^{p}\right]^{\frac{1}{p}}\right)\right] \mathcal{W}(x) x^{p-1} d x \\
& +\int_{u}^{v}\left(x^{p}-u^{p}\right)^{\alpha-1}[\underline{f}(x), \bar{f}(x)] \mathcal{W}(x) x^{p-1} d x, \\
& \quad=\frac{p}{\left(v^{p}-u^{p}\right)^{\alpha}} \int_{u}^{v}\left(x^{p}-u^{p}\right)^{\alpha-1}[\underline{f}(x), \bar{f}(x)] \mathcal{W}\left(\left[u^{p}-v^{p}-x^{p}\right]^{\frac{1}{p}}\right) x^{p-1} d x \\
& +\int_{u}^{v}\left(x^{p}-u^{p}\right)^{\alpha-1}[\underline{f}(x), \bar{f}(x)] \mathcal{W}(x) x^{p-1} d x, \\
& =\frac{p}{\left(v^{p}-u^{p}\right)^{\alpha}} \int_{u}^{v}\left(x^{p}-u^{p}\right)^{\alpha-1}[\underline{f}(x), \bar{f}(x)] \mathcal{W}(x) x^{p-1} d x \\
& +\int_{u}^{v}\left(x^{p}-u^{p}\right)^{\alpha-1}[\underline{f}(x), \bar{f}(x)] \mathcal{W}(x) x^{p-1} d x, \\
& =\frac{p}{\left(v^{p}-u^{p}\right)^{\alpha}}\left[\int_{u}^{v}\left(v^{p}-x^{p}\right)^{\alpha-1} f(x) \mathcal{W}(x) x^{p-1} d x+\int_{u}^{v}\left(x^{p}-u^{p}\right)^{\alpha-1} f(x) \mathcal{W}(x) x^{p-1} d x\right] .
\end{aligned}
$$

Therefore, we have

$$
\begin{gathered}
\frac{p^{\alpha} \Gamma(\alpha)}{\left(v^{p}-u^{p}\right)^{\alpha}} f\left(\left[\frac{u^{p}+v^{p}}{2}\right]^{\frac{1}{p}}\right)\left[\mathcal{I}_{u^{+}}^{p, \alpha} \mathcal{W}(v)+\mathcal{I}_{v^{-}}^{p, \alpha} \mathcal{W}(u)\right] \\
\leq_{p} \frac{p^{\alpha} \Gamma(\alpha)}{\left(v^{p}-u^{p}\right)^{\alpha}}\left[\mathcal{I}_{u^{+}}^{p, \alpha} f \mathcal{W}(v)+\mathcal{I}_{v^{-}}^{p, \alpha} f \mathcal{W}(u)\right] .
\end{gathered}
$$

Now taking the multiplication of $(23)$ by $\varrho^{\alpha-1} \mathcal{W}\left(\left[\varrho v^{p}+(1-\varrho) u^{p}\right]^{\frac{1}{p}}\right)$, and integrating it with respect to $\varrho$ over $[0,1]$, we get

$$
\begin{aligned}
& \int_{0}^{1} \varrho^{\alpha-1} \mathcal{W}\left(\left[\varrho v^{p}+(1-\varrho) u^{p}\right]^{\frac{1}{p}}\right) f\left(\left[\varrho u^{p}+(1-\varrho) v^{p}\right]^{\frac{1}{p}}\right) d \varrho \\
& +\int_{0}^{1} \varrho^{\alpha-1} \mathcal{W}\left(\left[\varrho v^{p}+(1-\varrho) u^{p}\right]^{\frac{1}{p}}\right) f\left(\left[\varrho v^{p}+(1-\varrho) u^{p}\right]^{\frac{1}{p}}\right) d \varrho \\
& \quad \leq_{p}[f(u)+f(v)] \int_{0}^{1} \varrho^{\alpha-1} \mathcal{W}\left(\left[\varrho v^{p}+(1-\varrho) u^{p}\right]^{\frac{1}{p}}\right) d \varrho .
\end{aligned}
$$

Therefore, we have

$$
\frac{p^{\alpha} \Gamma(\alpha)}{\left(v^{p}-u^{p}\right)^{\alpha}}\left[\mathcal{I}_{u^{+}}^{p, \alpha} f \mathcal{W}(v) \widetilde{+} \mathcal{I}_{v^{-}}^{p, \alpha} f \mathcal{W}(u)\right] \leq_{p} \frac{p^{\alpha} \Gamma(\alpha)}{\left(v^{p}-u^{p}\right)^{\alpha}} \cdot \frac{\mathcal{F}(u) \widetilde{+} \mathcal{F}(v)}{2}\left[\mathcal{I}_{u^{+}}^{p, \alpha} \mathcal{W}(v)+\mathcal{I}_{v^{-}}^{p, \alpha} \mathcal{W}(u)\right]
$$

Combining (20) and (21), we get

$$
\begin{aligned}
& f\left(\left[\frac{u^{p}+v^{p}}{2}\right]^{\frac{1}{p}}\right)\left[\mathcal{I}_{u^{+}}^{p, \alpha} \mathcal{W}(v)+\mathcal{I}_{v^{-}}^{p, \alpha} \mathcal{W}(u)\right] \\
& \leq_{p}\left[\mathcal{I}_{u^{+}}^{p, \alpha} \mathcal{W}(v)+\mathcal{I}_{v^{-}}^{p, \alpha} f \mathcal{W}(u)\right] \leq_{p} \frac{f(u)+f(v)}{2}\left[\mathcal{I}_{u^{+}}^{p, \alpha} \mathcal{W}(v)+\mathcal{I}_{v^{-}}^{p, \alpha} \mathcal{W}(u)\right]
\end{aligned}
$$

and the theorem has been proved.

Remark 5. Let $p=1$. Then, Theorem 4 reduces to the result for LR-convex-IVF, which is also a new one:

$f\left(\frac{u+v}{2}\right)\left[\mathcal{I}_{u^{+}}^{\alpha} \mathcal{W}(v)+\mathcal{I}_{v^{-}}^{\alpha} \mathcal{W}(u)\right] \leq_{p}\left[\mathcal{I}_{u^{+}}^{\alpha} f \mathcal{W}(v)+\mathcal{I}_{v^{-}}^{\alpha} f \mathcal{W}(u)\right] \leq_{p} \frac{f(u)+f(v)}{2}\left[\mathcal{I}_{u^{+}}^{\alpha} \mathcal{W}(v)+\mathcal{I}_{v^{-}}^{\alpha} \mathcal{W}(u)\right]$ 
Let $\alpha=1$. Then, Theorem 4 reduces to the result for LR-p-convex-IVF, which is also a new one:

$$
f\left(\left[\frac{u^{p}+v^{p}}{2}\right]^{\frac{1}{p}}\right) \leq_{p} \frac{1}{\int_{u}^{v} x^{p-1} \mathcal{W}(x) d x} \int_{u}^{v} x^{p-1} f(x) \mathcal{W}(x) d x \leq_{p} \frac{f(u)+f(v)}{2}
$$

Let $p=\alpha=1$. Then, Theorem 4 reduces to the result for LR-convex-IVF, which is also a new one:

$$
f\left(\frac{u+v}{2}\right) \leq_{p} \frac{1}{\int_{u}^{v} \mathcal{W}(x) d x} \int_{u}^{v} f(x) \mathcal{W}(x) d x \leq_{p} \frac{f(u)+f(v)}{2}
$$

If $f=\bar{f}$ and $\alpha=1$, then from Theorem 4, we get Theorem 5 of [39].

If $f=\bar{f}$ and $\alpha=1$, then from Theorem 4 , we obtain the classical HH-Fejér type inequality (2).

If $f=\bar{f}$ and $\mathcal{W}(x)=p=\alpha=1$, then from Theorem 4 , we get the classical $H H$ inequality (1).

If $\mathcal{W}(x)=1$, then from Theorem 4 , we get Theorem 3 .

Theorem 5. Let $p, \alpha>0, u, v \in I$ with $v>u$ and $f, g \in \Im \mathcal{L}_{([u, v])}$. If $f, g \in \operatorname{LRSX}\left([u, v], \mathbb{R}_{I}^{+}, p\right)$, then we have

$$
\frac{p^{\alpha} \Gamma(\alpha)}{2\left(v^{p}-u^{p}\right)^{\alpha}}\left[\mathcal{I}_{u^{+}}^{p, \alpha} f(v) g(v)+\mathcal{I}_{v^{-}}^{p, \alpha} f(u) f(u)\right] \leq_{p}\left(\frac{1}{2}-\frac{\alpha}{(\alpha+1)(\alpha+2)}\right) M(u, v)+\left(\frac{\alpha}{(\alpha+1)(\alpha+2)}\right) N(u, v) .
$$

$$
\text { If } f, g \in \operatorname{LRSV}\left([u, v], \mathbb{R}_{I}^{+}, p\right) \text {, then }
$$

$$
\frac{p^{\alpha} \Gamma(\alpha)}{2\left(v^{p}-u^{p}\right)^{\alpha}}\left[\mathcal{I}_{u^{+}}^{p, \alpha} f(v) f(v)+\mathcal{I}_{v^{-}}^{p, \alpha} f(u) f(u)\right] \geq_{p}\left(\frac{1}{2}-\frac{\alpha}{(\alpha+1)(\alpha+2)}\right) M(u, v)+\left(\frac{\alpha}{(\alpha+1)(\alpha+2)}\right) N(u, v)
$$

where

$$
M(u, v)=[f(u) g(u)+f(v) g(v)]
$$

and

$$
N(u, v)=[f(u) g(v)+f(v) g(u)]
$$

Proof. Since $f, g \in \operatorname{LRSX}\left([u, v], \mathbb{R}_{I}^{+}, p\right)$, then for $\varrho \in[0,1]$ we have

$$
f\left(\left[\varrho u^{p}+(1-\varrho) v^{p}\right]^{\frac{1}{p}}\right) \leq_{p} \varrho f(u)+(1-\varrho) f(v)
$$

and

$$
g\left(\left[\varrho u^{p}+(1-\varrho) v^{p}\right]^{\frac{1}{p}}\right) \leq_{p} \varrho g(u)+(1-\varrho) g(v) .
$$
we have

From the definition of $p$-convex-IVFs, it follows that $0 \leq_{p} f(x)$ and $0 \leq_{p} g(x)$, then

$$
\begin{aligned}
& f\left(\left[\varrho u^{p}+(1-\varrho) v^{p}\right]^{\frac{1}{p}}\right) g\left(\left[\varrho u^{p}+(1-\varrho) v^{p}\right]^{\frac{1}{p}}\right) \\
& \leq_{p} \varrho^{2} f(u) g(u)+(1-\varrho)^{2} f(v) g(v)+\varrho(1-\varrho)[f(v) g(u)+f(u) g(v)]
\end{aligned}
$$

Similarly, we have

$$
\begin{aligned}
& f\left(\left[(1-\varrho) u^{p}+\varrho v^{p}\right]^{\frac{1}{p}}\right) g\left(\left[(1-\varrho) u^{p}+\varrho v^{p}\right]^{\frac{1}{p}}\right) \\
& \leq_{p}(1-\varrho)^{2} g(u) f(u)+\varrho^{2} f(v) g(v)+\varrho(1-\varrho)[g(v) f(u)+g(u) f(v)]
\end{aligned}
$$


Adding (32) and (33), we get

$$
\begin{aligned}
& f\left(\left[\varrho u^{p}+(1-\varrho) v^{p}\right]^{\frac{1}{p}}\right) g\left(\left[\varrho u^{p}+(1-\varrho) v^{p}\right]^{\frac{1}{p}}\right) \\
& +f\left(\left[(1-\varrho) u^{p}+\varrho v^{p}\right]^{\frac{1}{p}}\right) g\left(\left[(1-\varrho) u^{p}+\varrho v^{p}\right]^{\frac{1}{p}}\right) \\
& \leq_{p}\left[\varrho^{2}+(1-\varrho)^{2}\right][f(u) g(u)+f(v) g(v)]+2 \varrho(1-\varrho)[f(v) g(u)+f(u) g(v)]
\end{aligned}
$$

Multiplying both sides of (34) by $\varrho^{\alpha-1}$ and integrating the obtained result with respect to $\varrho$ over $(0,1)$, we have

$$
\begin{aligned}
& \int_{0}^{1} \varrho^{\alpha-1} f\left(\left[\varrho u^{p}+(1-\varrho) v^{p}\right]^{\frac{1}{p}}\right) g\left(\left[\varrho u^{p}+(1-\varrho) v^{p}\right]^{\frac{1}{p}}\right) d \varrho \\
& +\int_{0}^{1} \varrho^{\alpha-1} f\left(\left[(1-\varrho) u^{p}+\varrho v^{p}\right]^{\frac{1}{p}}\right) g\left(\left[(1-\varrho) u^{p}+\varrho v^{p}\right]^{\frac{1}{p}}\right) d \varrho \\
& \leq_{p} M(u, v) \int_{0}^{1} \varrho^{\alpha-1}\left[\varrho^{2}+(1-\varrho)^{2}\right]+2 N(u, v) \int_{0}^{1} \varrho^{\alpha-1} \varrho(1-\varrho) d \varrho .
\end{aligned}
$$

Form (35), we have

$$
\begin{aligned}
& \int_{0}^{1} \varrho^{\alpha-1} f\left(\left[\varrho u^{p}+(1-\varrho) v^{p}\right]^{\frac{1}{p}}\right) g\left(\left[\varrho u^{p}+(1-\varrho) v^{p}\right]^{\frac{1}{p}}\right) d \varrho \\
& +\int_{0}^{1} \varrho^{\alpha-1} f\left(\left[(1-\varrho) u^{p}+\varrho v^{p}\right]^{\frac{1}{p}}\right) g\left(\left[(1-\varrho) u^{p}+\varrho v^{p}\right]^{\frac{1}{p}}\right) d \varrho \\
& =\frac{p^{\alpha} \Gamma(\alpha)}{\left(v^{p}-u^{p}\right)^{\alpha}}\left[\mathcal{I}_{u^{+}}^{p, \alpha} f(v) g(v)+\mathcal{I}_{v^{-}}^{p, \alpha} f(u) g(u)\right] .
\end{aligned}
$$

and

$$
\begin{gathered}
M(u, v) \int_{0}^{1} \varrho^{\alpha-1}\left[\varrho^{2}+(1-\varrho)^{2}\right]+2 N(u, v) \int_{0}^{1} \varrho^{\alpha-1} \varrho(1-\varrho) d \varrho \\
=\frac{2}{\alpha}\left(\frac{1}{2}-\frac{\alpha}{(\alpha+1)(\alpha+2)}\right) M(u, v)+\frac{2}{\alpha}\left(\frac{\alpha}{(\alpha+1)(\alpha+2)}\right) N(u, v) .
\end{gathered}
$$

From (36) and (37), we have

$$
\frac{p^{\alpha} \Gamma(\alpha)}{2\left(v^{p}-u^{p}\right)^{\alpha}}\left[\mathcal{I}_{u^{+}}^{p, \alpha} f(v) g(v)+\mathcal{I}_{v^{-}}^{p, \alpha} f(u) f(u)\right] \leq_{p}\left(\frac{1}{2}-\frac{\alpha}{(\alpha+1)(\alpha+2)}\right) M(u, v)+\left(\frac{\alpha}{(\alpha+1)(\alpha+2)}\right) N(u, v)
$$

and the required result has been obtained.

Example 3. Let $p$ be an odd number, $[u, v]=[0,2], \alpha=\frac{1}{2}, f(x)=\left[e^{x^{p}}-4,2 x^{p}\right]$, and $g(x)=\left[x^{p}-3,2 x^{p}\right]$. Then, $f g \in \Im \mathcal{L}_{([u, v])}$ and

$$
\begin{aligned}
& \frac{p^{\alpha} \Gamma(1+\alpha)}{2\left(v^{p}-u^{p}\right)^{\alpha}}\left[\mathcal{I}_{u^{+}}^{p, \alpha} f(v) g(v)+\mathcal{I}_{v^{-}}^{p, \alpha} f(u) g(u)\right] \\
& =\frac{\Gamma\left(\frac{3}{2}\right)}{2 \sqrt{2}} \frac{1}{\sqrt{\pi}} \int_{0}^{2}\left(2^{p}-x^{p}\right)^{\frac{-1}{2}} x^{p-1}\left[\left(4-e^{x^{p}}\right)\left(3-x^{p}\right), 4 x^{2 p}\right] d x+\frac{\Gamma\left(\frac{3}{2}\right)}{2 \sqrt{2}} \frac{1}{\sqrt{\pi}} \int_{0}^{2}\left(x^{p}\right)^{\frac{-1}{2}} x^{p-1}\left[\left(4-e^{x^{p}}\right)\left(3-x^{p}\right), 4 x^{2 p}\right] d x \\
& \approx[2.6446,5.8664] .
\end{aligned}
$$

Note that

$$
\begin{aligned}
& M(u, v)=[f(u) g(u)+f(v) g(v)]=\left[13-e^{2}, 16\right] \\
& N(u, v)=[f(u) g(v)+f(v) g(u)]=\left[15-3 e^{2}, 0\right] .
\end{aligned}
$$


Therefore, we have

$$
\begin{gathered}
\left(\frac{1}{2}-\frac{\alpha}{(\alpha+1)(\alpha+2)}\right) M(u, v)+\left(\frac{\alpha}{(\alpha+1)(\alpha+2)}\right) N(u, v)=\frac{11}{15}\left[13-e^{2}, 16\right]+\frac{2}{15}\left[15-3 e^{2}, 0\right] \\
\approx[3.1591,11.7333] .
\end{gathered}
$$

It follows that

$$
[2.6446,5.8664] \leq_{p}[3.1591,11.7333],
$$

and Theorem 5 has been illustrated.

Theorem 6. Let $p, \alpha>0, u, v \in I$ with $v>u$ and $f, g \in \Im \mathcal{L}_{([u, v])}$. If $f, g \in \operatorname{LRSX}\left([u, v], \mathbb{R}_{I}^{+}, p\right)$, then we have

$$
\begin{gathered}
2 f\left(\left[\frac{u^{p}+v^{p}}{2}\right]^{\frac{1}{p}}\right) g\left(\left[\frac{u^{p}+v^{p}}{2}\right]^{\frac{1}{p}}\right) \leq_{p} \frac{p^{\alpha} \Gamma(\alpha+1)}{2\left(v^{p}-u^{p}\right)^{\alpha}}\left[\mathcal{I}_{u^{+}}^{p, \alpha} f(v) g(v)+\mathcal{I}_{v^{-}}^{p, \alpha} f(u) g(u)\right] \\
+\left(\frac{1}{2}-\frac{\alpha}{(\alpha+1)(\alpha+2)}\right) N(u, v)+\left(\frac{\alpha}{(\alpha+1)(\alpha+2)}\right) M(u, v) .
\end{gathered}
$$

If $f, g \in \operatorname{LRSV}\left([u, v], \mathbb{R}_{I}^{+}, p\right)$, then

$$
\begin{gathered}
f\left(\left[\frac{u^{p}+v^{p}}{2}\right]^{\frac{1}{p}}\right) g\left(\left[\frac{u^{p}+v^{p}}{2}\right]^{\frac{1}{p}}\right) \geq_{p} \frac{p^{\alpha} \Gamma(\alpha+1)}{4\left(v^{p}-u^{p}\right)^{\alpha}}\left[\mathcal{I}_{u^{+}}^{p, \alpha} f(v) g(v)+\mathcal{I}_{v^{-}}^{p, \alpha} f(u) g(u)\right] \\
+\frac{1}{2}\left(\frac{1}{2}-\frac{\alpha}{(\alpha+1)(\alpha+2)}\right) N(u, v)+\frac{1}{2}\left(\frac{\alpha}{(\alpha+1)(\alpha+2)}\right) M(u, v)
\end{gathered}
$$

where $M(u, v)$ and $N(u, v)$ are given in Theorem 5 .

Proof. Since $f, g \in \operatorname{LRSX}\left([u, v], \mathbb{R}_{I}^{+}, p\right)$, then by hypothesis, for $\varrho \in[0,1]$ we have

$$
\begin{aligned}
& f\left(\left[\frac{u^{p}+v^{p}}{2}\right]^{\frac{1}{p}}\right) g\left(\left[\frac{u^{p}+v^{p}}{2}\right]^{\frac{1}{p}}\right) \\
& =f\left[\frac{\left[(1-\varrho) u^{p}+\varrho v^{p}\right]^{\frac{1}{p}}}{2}+\frac{\left[\varrho u^{p}+(1-\varrho) v^{p}\right]^{\frac{1}{p}}}{2}\right] \times g\left[\frac{\left[(1-\varrho) u^{p}+\varrho v^{p}\right]^{\frac{1}{p}}}{2}+\frac{\left[\varrho u^{p}+(1-\varrho) v^{p}\right]^{\frac{1}{p}}}{2}\right] \\
& \leq_{p} \frac{1}{4}\left[f\left(\left[\varrho u^{p}+(1-\varrho) v^{p}\right]^{\frac{1}{p}}\right)+f\left(\left[(1-\varrho) u^{p}+\varrho v^{p}\right]^{\frac{1}{p}}\right)\right] \\
& \times\left[g\left(\left[\varrho u^{p}+(1-\varrho) v^{p}\right]^{\frac{1}{p}}\right)+g\left(\left[(1-\varrho) u^{p}+\varrho v^{p}\right]^{\frac{1}{p}}\right)\right] \\
& =\frac{1}{4}\left[f\left(\left[\varrho u^{p}+(1-\varrho) v^{p}\right]^{\frac{1}{p}}\right) g\left(\left[\varrho u^{p}+(1-\varrho) v^{p}\right]^{\frac{1}{p}}\right)\right] \\
& +\left[f\left(\left[(1-\varrho) u^{p}+\varrho v^{p}\right]^{\frac{1}{p}}\right) g\left(\left[(1-\varrho) u^{p}+\varrho v^{p}\right]^{\frac{1}{p}}\right)\right] \\
& +\left[g\left(\left[(1-\varrho) u^{p}+\varrho v^{p}\right]^{\frac{1}{p}}\right) f\left(\left[\varrho u^{p}+(1-\varrho) v^{p}\right]^{\frac{1}{p}}\right)\right] \\
& +\left[f\left(\left[(1-\varrho) u^{p}+\varrho v^{p}\right]^{\frac{1}{p}}\right) g\left(\left[\varrho u^{p}+(1-\varrho) v^{p}\right]^{\frac{1}{p}}\right)\right] \\
& \leq_{p} \frac{1}{4}\left[f\left(\left[\varrho u^{p}+(1-\varrho) v^{p}\right]^{\frac{1}{p}}\right)+g\left(\left[\varrho u^{p}+(1-\varrho) v^{p}\right]^{\frac{1}{p}}\right)\right. \\
& \left.+f\left(\left[(1-\varrho) u^{p}+\varrho v^{p}\right]^{\frac{1}{p}}\right) g\left(\left[(1-\varrho) u^{p}+\varrho v^{p}\right]^{\frac{1}{p}}\right)\right] \\
& +\frac{1}{4}\left(2 \varrho^{2}-2 \varrho+1\right) N(u, v)+\frac{1}{2} \varrho(1-\varrho) M(u, v) .
\end{aligned}
$$


Taking both multiplications of (40) with $\varrho^{\alpha-1}$ and integrating the result with respect to over $(0,1)$, we have

$$
\begin{gathered}
\int_{0}^{1} \varrho^{\alpha-1} f\left(\left[\frac{u^{p}+v^{p}}{2}\right]^{\frac{1}{p}}\right) g\left(\left[\frac{u^{p}+v^{p}}{2}\right]^{\frac{1}{p}}\right) d \varrho \\
\leq p \frac{1}{4}\left[\int_{0}^{1} \varrho^{\alpha-1} f\left(\left[\varrho u^{p}+(1-\varrho) v^{p}\right]^{\frac{1}{p}}\right) g\left(\left[\varrho u^{p}+(1-\varrho) v^{p}\right]^{\frac{1}{p}}\right) d \varrho\right. \\
\left.+\int_{0}^{1} \varrho^{\alpha-1} f\left(\left[(1-\varrho) u^{p}+\varrho v^{p}\right]^{\frac{1}{p}}\right) g\left(\left[(1-\varrho) u^{p}+\varrho v^{p}\right]^{\frac{1}{p}}\right) d \varrho\right] \\
+\frac{1}{4} \int_{0}^{1} \varrho^{\alpha-1}\left(2 \varrho^{2}-2 \varrho+1\right) N(u, v)+\frac{1}{2} \int_{0}^{1} \varrho^{\alpha-1} \varrho(1-\varrho) M(u, v) d \varrho .
\end{gathered}
$$

From (41), we get

$$
\begin{aligned}
& \int_{0}^{1} \varrho^{\alpha-1} f\left(\left[\frac{u^{p}+v^{p}}{2}\right]^{\frac{1}{p}}\right) g\left(\left[\frac{u^{p}+v^{p}}{2}\right]^{\frac{1}{p}}\right) d \varrho \\
& =\left[\int_{0}^{1} \varrho^{\alpha-1} f\left(\left[\frac{u^{p}+v^{p}}{2}\right]^{\frac{1}{p}}\right) \underline{g}\left(\left[\frac{u^{p}+v^{p}}{2}\right]^{\frac{1}{p}}\right) d \varrho, \int_{0}^{1} \varrho^{\alpha-1} \bar{f}\left(\left[\frac{u^{p}+v^{p}}{2}\right]^{\frac{1}{p}}\right) \bar{g}\left(\left[\frac{u^{p}+v^{p}}{2}\right]^{\frac{1}{p}}\right) d \varrho\right] \\
& =\left[\frac{1}{\alpha} f\left(\left[\frac{u^{p}+v^{p}}{2}\right]^{\frac{1}{p}}\right) g\left(\left[\frac{u^{p}+v^{p}}{2}\right]^{\frac{1}{p}}\right), \frac{1}{\alpha} \bar{f}\left(\left[\frac{u^{p}+v^{p}}{2}\right]^{\frac{1}{p}}\right) \bar{g}\left(\left[\frac{u^{p}+v^{p}}{2}\right]^{\frac{1}{p}}\right)\right] \\
& =\frac{1}{\alpha} f\left(\left[\frac{u^{p}+v^{p}}{2}\right]^{\frac{1}{p}}\right) g\left(\left[\frac{u^{p}+v^{p}}{2}\right]^{\frac{1}{p}}\right) .
\end{aligned}
$$

On the other hand, from (42) and taking $\varrho x^{p}=\varrho u^{p}+(1-\varrho) v^{p}$ and $y^{p}=(1-\varrho) u^{p}+$ $\varrho v^{p}$, we get

$$
\begin{aligned}
& \frac{1}{4}\left[\int_{0}^{1} \varrho^{\alpha-1} f\left(\left[\varrho u^{p}+(1-\varrho) v^{p}\right]^{\frac{1}{p}}\right) g\left(\left[\varrho u^{p}+(1-\varrho) v^{p}\right]^{\frac{1}{p}}\right) d \varrho\right. \\
& \left.\quad+\int_{0}^{1} \varrho^{\alpha-1} f\left(\left[(1-\varrho) u^{p}+\varrho v^{p}\right]^{\frac{1}{p}}\right) g\left(\left[(1-\varrho) u^{p}+\varrho v^{p}\right]^{\frac{1}{p}}\right) d \varrho\right] \\
& \quad+\frac{1}{4} \int_{0}^{1} \varrho^{\alpha-1}\left(2 \varrho^{2}-2 \varrho+1\right) N(u, v) d \varrho+\frac{1}{2} \int_{0}^{1} \varrho^{\alpha-1} \varrho(1-\varrho) M(u, v) d \varrho
\end{aligned}
$$$$
=\frac{p}{4\left(v^{p}-u^{p}\right)^{\alpha}}\left[\begin{array}{c}
\int_{u}^{v}\left(v^{p}-x^{p}\right)^{\alpha-1} \underline{f}(x) \underline{g}(x) x^{p-1} d x+\int_{u}^{v}\left(y^{p}-u^{p}\right)^{\alpha-1} \underline{f}(y) \underline{g}(y) y^{p-1} d y, \\
\int_{0}^{1}\left(v^{p}-x^{p}\right)^{\alpha-1} \bar{f}(x) \bar{g}(x) x^{p-1} d x+\int_{u}^{v}\left(y^{p}-u^{p}\right)^{\alpha-1} \bar{f}(y) \bar{g}(y) y^{p-1} d y
\end{array}\right]
$$

$+\frac{1}{2 \alpha}\left(\frac{1}{2}-\frac{\alpha}{(\alpha+1)(\alpha+2)}\right) N(u, v)+\frac{1}{2 \alpha}\left(\frac{\alpha}{(\alpha+1)(\alpha+2)}\right) M(u, v)$

$=\frac{p^{\alpha} \Gamma(\alpha+1)}{4\left(v^{p}-u^{p}\right)^{\alpha}}\left[\mathcal{I}_{u^{+}}^{p, \alpha} f(v) g(v)+\mathcal{I}_{v^{-}}^{p, \alpha} f(u) g(u)\right]$

$+\frac{1}{2 \alpha}\left(\frac{1}{2}-\frac{\alpha}{(\alpha+1)(\alpha+2)}\right) N(u, v)+\frac{1}{2 \alpha}\left(\frac{\alpha}{(\alpha+1)(\alpha+2)}\right) M(u, v)$.

From (42) and (43), (41) becomes

$$
\begin{gathered}
2 f\left(\left[\frac{u^{p}+v^{p}}{2}\right]^{\frac{1}{p}}\right) g\left(\left[\frac{u^{p}+v^{p}}{2}\right]^{\frac{1}{p}}\right) \leq_{p} \frac{p^{\alpha} \Gamma(\alpha+1)}{2\left(v^{p}-u^{p}\right)^{\alpha}}\left[\mathcal{I}_{u^{+}}^{p, \alpha} f(v) g(v)+\mathcal{I}_{v^{-}}^{p, \alpha} f(u) g(u)\right] \\
+\left(\frac{1}{2}-\frac{\alpha}{(\alpha+1)(\alpha+2)}\right) N(u, v)+\left(\frac{\alpha}{(\alpha+1)(\alpha+2)}\right) M(u, v)
\end{gathered}
$$

Hence, Theorem 6 has been proved.

Example 4. Let $p$ be an odd number and $\alpha=1$ for $\varrho \in[0,1]$, and the $L R$-p-convex $f:[u, \vartheta]=$ $[2,3] \rightarrow \mathbb{R}_{I}^{+}$and LR-p-convex IVFs $g:[u, \vartheta]=[2,3] \rightarrow \mathbb{R}_{I}^{+}$are respectively defined by $f(x)=$ 
$\left[2-x^{\frac{p}{2}}, 2\left(2-x^{\frac{p}{2}}\right)\right]$ and $g(x)=\left[x^{p}, 2 x^{p}\right]$. Since $f_{*}(x)=2-x^{\frac{p}{2}}, f^{*}(x)=2\left(2-x^{\frac{p}{2}}\right)$ and $g_{*}(x)=x^{p}, g^{*}(x)=2 x^{p}$, then we compute the following

$$
\begin{gathered}
2 f_{*}\left(\left[\frac{u^{p}+\vartheta^{p}}{2}\right]^{\frac{1}{p}}\right) \times g_{*}\left(\left[\frac{u^{p}+\vartheta^{p}}{2}\right]^{\frac{1}{p}}\right)=\frac{20-5 \sqrt{10}}{2} \\
2 f^{*}\left(\left[\frac{u^{p}+\vartheta^{p}}{2}\right]^{\frac{1}{p}}\right) \times g^{*}\left(\left[\frac{u^{p}+\vartheta^{p}}{2}\right]^{\frac{1}{p}}\right)=40-10 \sqrt{10}, \\
\frac{p^{\alpha} \Gamma(\alpha+1)}{2\left(v^{p}-u^{p}\right)^{\alpha}}\left[\mathcal{I}_{u^{p}}^{p, \alpha} f_{*}(v) \times g_{*}(v)+\mathcal{I}_{v^{p}}^{p, \alpha} f_{*}(u) \times g_{*}(u)\right]=1 \\
\frac{p^{\alpha} \Gamma(\alpha+1)}{2\left(v^{p}-u^{p}\right)^{\alpha}}\left[\mathcal{I}_{u^{p}}^{p, \alpha} f^{*}(v) \times g^{*}(v)+\mathcal{I}_{v^{p}}^{p, \alpha} f^{*}(u) \times g^{*}(u)\right]=4, \\
\left(\frac{\alpha}{(\alpha+1)(\alpha+2)}\right) \mathcal{M}_{*}(u, \vartheta)=\frac{1}{6}(10-2 \sqrt{2}-3 \sqrt{3}) \\
\left(\frac{\alpha}{(\alpha+1)(\alpha+2)}\right) \mathcal{M}^{*}(u, \vartheta)=\frac{4}{6}(10-2 \sqrt{2}-3 \sqrt{3}), \\
\frac{1}{2}-\frac{\alpha}{(\alpha+1)(\alpha+2)} \mathcal{N}_{*}(u, \vartheta)=\frac{1}{3}(10-3 \sqrt{2}-2 \sqrt{3}) \\
\frac{1}{2}-\frac{\alpha}{(\alpha+1)(\alpha+2)} \mathcal{N}^{*}(u, \vartheta)=\frac{4}{3}(10-3 \sqrt{2}-2 \sqrt{3}),
\end{gathered}
$$

that means

$$
\begin{gathered}
\frac{20-5 \sqrt{10}}{2} \leq\left(1+\frac{30-8 \sqrt{2}-7 \sqrt{3}}{6}\right), \\
40-10 \sqrt{10} \leq\left(4+\frac{60-16 \sqrt{2}-14 \sqrt{3}}{3}\right),
\end{gathered}
$$

hence, Theorem 6 has been illustrated.

\section{Conclusions}

In this work, we introduced the new class of LR-p-convex interval-valued functions and established some new Hermite-Hadamard inequalities by means of the pseudo order relation via Katugampola fractional integral operator. Useful examples that verify the applicability of the theory developed in this study are presented. We intend to use various types of LR-convex interval-valued functions to construct interval inequalities of intervalvalued functions. In the future, we will try to explore this concept for fuzzy-interval-valued functions by means of the fuzzy pseudo order relation.

Author Contributions: Conceptualization, M.B.K. and M.A.N.; validation, P.O.M., D.B. and J.L.G.G.; formal analysis, D.B. and J.L.G.G.; investigation, M.B.K., M.A.N. and D.B.; resources, M.B.K. and M.A.N.; writing—original draft, M.B.K. and M.A.N.; writing—review and editing, M.B.K., P.O.M. and D.B.; visualization, M.A.N., P.O.M. and D.B.; supervision, M.A.N. and P.O.M.; project administration, M.A.N. and J.L.G.G. All authors have read and agreed to the published version of the manuscript.

Data Availability Statement: No data were used to support this study.

Acknowledgments: The authors would like to thank the Rector, COMSATS University Islamabad, Islamabad, Pakistan, for providing excellent research and academic environments. This work has been partially supported by Ministerio de Ciencia, Innovaci ón y Universidades, grant number PGC2018-097198-B-I00 and by Fundaci ón Séneca of Región de Murcia, grant number 20783/PI/18.

Conflicts of Interest: The authors declare no conflict of interest.

\section{References}

1. Hermite, C. Sur deux limites d'une intégrale définie. Mathesis 1883, 3, 82-97.

2. Hadamard, J. Étude sur les propriétés des fonctions entières et en particulier d'une fonction considérée par Riemann. J. Mathématiques Pures Appliquées 1893, 7, 171-215.

3. Awan, M.U.; Akhtar, N.; Iftikhar, S.; Noor, M.A.; Chu, Y.-M. New Hermite-Hadamard type inequalities for n-polynomial harmonically convex functions. J. Inequal. Appl. 2020, 2020, 125. [CrossRef]

4. Latif, M.A.; Rashid, S.; Dragomir, S.S.; Chu, Y.-M. Hermite-Hadamard type inequalities for co-ordinated convex and quasi-convex functions and their applications. J. Inequal. Appl. 2019, 2019, 317. [CrossRef] 
5. Chu, Y.-M.; Wang, G.-D.; Zhang, X.-H. The Schur multiplicative and harmonic convexities of the complete symmetric function. Math. Nachr. 2011, 284, 653-663. [CrossRef]

6. Chu, Y.M.; Xia, W.-F.; Zhang, X.-H. The Schur concavity, Schur multiplicative and harmonic convexities of the second dual form of the Hamy symmetric function with applications. J. Multivar. Anal. 2012, 105, 412-442. [CrossRef]

7. Zaheer Ullah, S.; Adil Khan, M.; Khan, Z.A.; Chu, Y.-M. Integral majorization type inequalities for the functions in the sense of strong convexity. J. Funct. Spaces 2019, 2019, 9487823. [CrossRef]

8. Zaheer Ullah, S.; Adil Khan, M.; Chu, Y.-M. Majorization theorems for strongly convex functions. J. Inequal. Appl. 2019, $2019,58$. [CrossRef]

9. Varošanec, S. On h-convexity. J. Math. Anal. Appl. 2007, 326, 303-311. [CrossRef]

10. Zhang, K.-S.; Wan, J.-P. p-convex functions and their properties. Pure Appl. Math. 2007, 23, 130-133.

11. Chang, S.S.; Zhu, Y.G. On variational inequalities for fuzzy mappings. Fuzzy Sets Syst. 1989, 32, 359-367. [CrossRef]

12. Nanda, S.; Kar, K. Convex fuzzy mappings. Fuzzy Sets Syst. 1992, 48, 129-132. [CrossRef]

13. Noor, M.A. Fuzzy preinvex functions. Fuzzy Sets Syst. 1994, 64, 95-104. [CrossRef]

14. Zaheer Ullah, S.; Adil Khan, M.; Chu, Y.-M. A note on generalized convex functions. J. Inequal. Appl. 2019, 2019, 15. [CrossRef]

15. Liu, W. New integral inequalities involving beta function via P-convexity. Miskolc. Math. Notes 2014, 15, 585-591. [CrossRef]

16. Fej'er, L. Uberdie Fourierreihen II. Math. Naturwise. Anz. Ungar. Akad. Wiss. 1906, 24, 369-390.

17. Breckner, W.W. Stetigkeitsaussagen für eine Klasse verallgemeinerter konvexer funktionen in topologischen linearen Räumen. Pupl. Inst. Math. 1978, 23, 13-20.

18. Hudzik, H.; Maligranda, L. Some remarks on s-convex functions. Aequat. Math. 1994, 48, 100-111. [CrossRef]

19. Iscan, I. Hermite-Hadamard type inequalities for p-convex functions. Int. J. Anal Appl. 2016, 11, 137-145.

20. Moore, R.E. Interval Analysis; Prentice Hall: Englewood Cliffs, NJ, USA, 1966.

21. Costa, T.M. Jensen's inequality type integral for fuzzy-interval-valued functions. Fuzzy Sets Syst. 2017, 327, 31-47. [CrossRef]

22. Costa, T.M.; Roman-Flores, H. Some integral inequalities for fuzzy-interval-valued functions. Inform. Sci. 2017, 420, 110-125. [CrossRef]

23. Román-Flores, H.; Chalco-Cano, Y.; Lodwick, W.A. Some integral inequalities for interval-valued functions. Comput. Appl. Math 2018, 37, 1306-1318. [CrossRef]

24. Roman-Flores, H.; Chalco-Cano, Y.; Silva, G.N. A note on Gronwall type inequality for interval-valued functions. In Proceedings of the IEEE IFSA World Congress and NAFIPS Annual Meeting, Edmonton, AB, Canada, 24-28 June 2013; Volume 35, pp. 1455-1458.

25. Chalco-Cano, Y.; Flores-Franulič, A.; Román-Flores, H. Ostrowski type inequalities for interval-valued functions using generalized Hukuhara derivative. Comput. Appl. Math. 2012, 31, 457-472.

26. Chalco-Cano, Y.; Lodwick, W.A.; Condori-Equice, W. Ostrowski type inequalities and applications in numerical integration for interval-valued functions. Soft Comput. 2015, 19, 3293-3300. [CrossRef]

27. Nikodem, K.; Snchez, J.L.; Snchez, L. Jensen and Hermite-Hadamard inequalities for strongly convex set-valued maps. Math. Aterna 2014, 4, 979-987.

28. Matkowski, J.; Nikodem, K. An integral Jensen inequality for convex multifunctions. Results Math. 1994, 26, 348-353. [CrossRef]

29. Zhang, D.; Guo, C.; Chen, D.; Wang, G. Jensen's inequalities for set-valued and fuzzy set-valued functions. Fuzzy Sets Syst. 2020, 2020, 1-27. [CrossRef]

30. Abdeljawad, T.; Baleanu, D. Monotonicity results for fractional difference operators with discrete exponential kernels. Adv. Differ. Equ. 2017, 2017, 78. [CrossRef]

31. Agarwal, R.; Purohit, S.D.; Kritika. A mathematical fractional model with nonsingular kernel for thrombin receptor activation in calcium signaling. Math. Methods Appl. Sci. 2019, 42, 7160-7171. [CrossRef]

32. Agarwal, R.; Yadav, M.P.; Baleanu, D.; Purohit, S.D. Existence and uniqueness of miscible flow equation through porous media with a non-singular fractional derivative. AIMS Math. 2019, 5, 1062-1073. [CrossRef]

33. Kumar, D.; Singh, J.; Purohit, S.D.; Swroop, R. A hybrid analytical algorithm for nonlinear fractional wave-like equations. Math. Model. Nat. Phenom. 2019, 14, 304. [CrossRef]

34. Budak, H.; Tunç, T.; Sarikaya, M.Z. Fractional Hermite-Hadamard type inequalities for interval-valued functions. Proc. Am. Math. Soc. 2019, 148, 705-718. [CrossRef]

35. Katugampola, U.N. A new approach to generalized fractional derivatives. Bull. Math. Anal. Appl. 2014, 6, 1-15.

36. Toplu, T.; Set, E.; İşcan, İ.; Maden, S. Hermite-Hadamard type inequalities for p-convex functions via Katugampola fractional integrals. Facta Univ. Ser. Math. Inform. 2019, 34, 149-164.

37. Adil Khan, M.; Begum, S.; Khurshid, Y.; Chu, Y.-M. Ostrowski type inequalities involving conformable fractional integrals. J. Inequal. Appl. 2018, 2018, 70. [CrossRef]

38. Fang, Z.B.; Shi, R. On the (p, h)-convex function and some integral inequalities. J. Inequal. Appl. 2014, 2014, 45. [CrossRef]

39. Kunt, M.; İşcan, İ. Hermite-Hadamard-Fejér type inequalities for p-convex functions. Arab J. Math. Sci. 2017, 23, 215-230. [CrossRef]

40. Liu, X.-L.; Ye, G.-J.; Zhao, D.-F.; Liu, W. Fractional Hermite-Hadamard type inequalities for interval-valued functions. J. Inequal. Appl. 2019, 2019, 26. [CrossRef]

41. Sarikaya, M.Z.; Set, E.; Yaldiz, H.; Başak, N. Hermite-Hadamard's inequalities for fractional integrals and related fractional inequalities. Math. Comput. Model. 2013, 57, 2403-2407. [CrossRef] 
42. Zhao, T.-H.; Chu, Y.-M.; Wang, H. Logarithmically complete monotonicity properties relating to the gamma function. Abstr. Appl. Anal. 2011, 2011, 896483. [CrossRef]

43. Precup, R.E.; Teban, T.A.; Albu, A.; Borlea, A.B.; Zamfirache, I.A.; Petriu, E.M. Evolving fuzzy models for prosthetic hand myoelectric-based control. IEEE Trans. Instrum. Meas. 2020, 69, 4625-4636. [CrossRef]

44. Khan, M.B.; Noor, M.A.; Noor, K.I.; Chu, Y.-M. New Hermite-Hadamard Type Inequalities for (h1, h2)-Convex Fuzzy-IntervalValued Functions. Adv. Differ. Equ. 2021, 2021, 6-20. [CrossRef]

45. Liu, P.; Khan, M.B.; Noor, M.A.; Noor, K.I. New Hermite-Hadamard and Jensen inequalities for log-s-convex fuzzy-intervalvalued functions in the second sense. Complex Intell. Syst. 2021, 2021, 1-15.

46. Khan, M.B.; Noor, M.A.; Abdullah, L.; Chu, Y.M. Some New Classes of Preinvex Fuzzy-Interval-Valued Functions and Inequalities. Int. J. Comput. Intell. Syst. 2021, 14, 1403-1418. [CrossRef]

47. Khan, M.B.; Mohammed, P.O.; Noor, M.A.; Hamed, Y.S. New Hermite-Hadamard inequalities in fuzzy-interval fractional calculus and related inequalities. Symmetry 2021, 13, 673. [CrossRef]

48. Liu, P.; Khan, M.B.; Noor, M.A.; Noor, K.I. On Strongly Generalized Preinvex Fuzzy Mappings. J. Math. 2021, 2021, 6657602. [CrossRef]

49. Khan, M.B.; Noor, M.A.; Noor, K.I.; Ab Ghani, A.T.; Abdullah, L. Extended perturbed mixed variational-like inequalities for fuzzy mappings. J. Math. 2021, 2021, 6652930. [CrossRef]

50. Khan, M.B.; Noor, M.A.; Noor, K.I.; Almusawa, H.; Nisar, K.S. Exponentially Preinvex Fuzzy Mappings and Fuzzy Exponentially Mixed Variational-Like Inequalities. Int. J. Anal. Appl. 2021, 19, 518-541.

51. Khan, M.B.; Noor, M.A.; Al-Bayatti, H.M.; Noor, K.I. Some New Inequalities for LR-Log-h-Convex Interval-Valued Functions by Means of Pseudo Order Relation. Appl. Math. 2021, 15, 459-470.

52. Sana, G.; Khan, M.B.; Noor, M.A.; Mohammed, P.O.; Chu, Y.M. Harmonically Convex Fuzzy-Interval-Valued Functions and Fuzzy-Interval Riemann-Liouville Fractional Integral Inequalities. Int. J. Comput. Intell. Syst. 2021, 14, 1809-1822. [CrossRef]

53. Khan, M.B.; Noor, M.A.; Noor, K.I.; Chu, Y.M. Higher-Order Strongly Preinvex Fuzzy Mappings and Fuzzy Mixed Variational-Like Inequalities. Int. J. Comput. Intell. Syst. 2021, 14, 1856-1870. [CrossRef]

54. Khan, M.B.; Noor, M.A.; Noor, K.I. On Some Characterization of Preinvex Fuzzy Mappings. Earth. J. Math. Sci. $2021,5,17-42$.

55. Khan, M.B.; Noor, M.A.; Noor, K.I. On Fuzzy Quasi-Invex Sets. Int. J. Algeb. Stat. 2021, 9, 11-26.

56. Mohammed, P.O. New generalized Riemann-Liouville fractional integral inequalities for convex functions. J. Math. Inequal. 2021, 15, 511-519. [CrossRef] 